\title{
An ab initio study on the ground and low-lying doublet electronic states of $\mathrm{SbO}_{2}$
}

\author{
Edmond P. F. Lee ${ }^{a), b)}$ \\ Department of Applied Biology and Chemical Technology, The Hong Kong Polytechnic University, \\ Hung Hom, Hong Kong \\ John M. Dyke \\ School of Chemistry, University of Southampton, Highfield, Southampton SO17 1BJ, United Kingdom \\ Foo-Tim Chau ${ }^{\mathrm{a}), \mathrm{c})}$ \\ Department of Applied Biology and Chemical Technology, The Hong Kong Polytechnic University, \\ Hung Hom, Hong Kong \\ Wan-Ki Chow \\ Department of Building Services Engineering, The Hong Kong Polytechnic University, \\ Hung Hom, Hong Kong \\ Daniel K. W. Mok \\ Department of Applied Biology and Chemical Technology, The Hong Kong Polytechnic University, \\ Hung Hom, Hong Kong
}

(Received 13 June 2006; accepted 13 July 2006; published online 11 August 2006)

\begin{abstract}
Geometry optimization and harmonic vibrational frequency calculations have been carried out on the low-lying doublet electronic states of antimony dioxide $\left(\mathrm{SbO}_{2}\right)$ employing a variety of ab initio methods, including the complete active space self-consistent field/multireference configuration interaction and the RCCSD(T) methods. Both large and small core relativistic effective core potentials were used for $\mathrm{Sb}$ in these calculations, together with valence basis sets of up to aug-cc-pV5Z quality. Contributions from outer core correlation and off-diagonal spin-orbit interaction to relative electronic energies have been calculated. The ground electronic state of $\mathrm{SbO}_{2}$ is determined to be the $\tilde{X}^{2} A_{1}$ state, as is the case for dioxides of other lighter group $15 p$-block (or group VA) elements. However, the $\widetilde{A}^{2} B_{2}$ and $\widetilde{B}^{2} A_{2}$ states are estimated to be only 4.1 and $10.7 \mathrm{kcal} / \mathrm{mole}$ above the $\tilde{X}^{2} A_{1}$ state, respectively, at the complete basis set limit. Reliable vertical excitation energies from the $\tilde{X}^{2} A_{1}$ state to low-lying excited states of $\mathrm{SbO}_{2}$ have been computed with a view to assist future spectral assignments of the absorption and/or laser-induced fluorescence spectra of $\mathrm{SbO}_{2}$, when they become available. () 2006 American Institute of Physics.
\end{abstract}

[DOI: $10.1063 / 1.2335445$ ]

\section{INTRODUCTION}

In recent work, clusters of antimony and antimony oxide have been studied in the gas phase using the technique of molecular beam mass spectrometry. ${ }^{1-5}$ In addition to investigating the physical and chemical properties of matter as it changes from atomic or molecular to the bulk via cluster formation, these studies also attempt to understand the complex mechanism of catalytic oxygen transfer on an antimony surface by using as a model the formation of antimony oxide clusters from the reaction between antimony metal clusters and molecular oxygen in the gas phase. The importance of antimony oxide in catalysis is well known (see, for example, Refs. 3 and 6-8), and there are various industrial applications of antimony oxide relating to its catalytic properties (see, for example, Refs. 3 and 9, and references therein),

\footnotetext{
a) Authors to whom correspondence should be addressed.

${ }^{b)}$ Also at School of Chemistry, University of Southampton. Electronic mail: epl@soton.ac.uk

${ }^{c)}$ Electronic mail: bcftchau@polyu.edu.hk
}

such as its use in antimony-halogen synergistic flame retardants in polymeric ${ }^{10-12}$ and nanocomposite ${ }^{13,14}$ materials. Unfortunately, the experimental technique of molecular beam mass spectrometry does not provide structural information (geometrical, molecular, and/or electronic) on the detected clusters. In this connection, density functional theory (DFT) and/or $a b$ initio calculations carried out on antimony oxide clusters would be invaluable in assisting the interpretation of, and the extraction of the maximum information from, the mass spectrometric results obtained. In fact, such calculations have been reported in some of the abovementioned mass spectrometric studies. ${ }^{3,5}$ In addition, DFT and/or $a b$ initio calculations have been performed in other spectroscopic studies of various antimony oxides in order to assist spectral assignments, ${ }^{9,15}$ and there are also numerous independent computational studies on antimony oxides, ${ }^{16-22}$ though most of these computational studies are on the diatomic antimony monoxide SbO. ${ }^{16-21}$ To our knowledge, however, there are only two computational studies, which have considered the triatomic antimony dioxide $\left(\mathrm{SbO}_{2}\right)$. 
TABLE I. Basis sets used for Sb.

\begin{tabular}{lllllll}
\hline \hline Basis & ECP $^{\mathrm{a}}$ & Valence set $^{\mathrm{a}}$ & Augmented $^{\mathrm{b}}$ & Frozen $^{\mathrm{c}}$ & Correlated $^{\mathrm{d}}$ & Nb $^{\mathrm{e}}$ \\
\hline A & ECP28MDF & Aug-cc-pVTZ & & $4 s 4 p 4 d$ & $5 s^{2} 5 p^{3}$ & 147 \\
B & ECP46MWB & Aug-cc-pVQZ & & $5 s^{2} 5 p^{3}$ & 239 \\
B1 & ECP46MWB & Aug-cc-pVQZ $(s p d f)^{\mathrm{f}}$ & & $5 s^{2} 5 p^{3}$ & 185 \\
C & ECP28MDF & Aug-cc-pV5Z & & $4 s 4 p 4 d$ & $5 s^{2} 5 p^{3}$ & 393 \\
D & ECP28MDF & Aug-cc-pV5Z & $3 d 2 f 2 g 2 h$ & $4 s 4 p$ & $4 d^{10} 5 s^{2} 5 p^{3}$ & 462 \\
E & ECP28MDF & Aug-cc-pV5Z & $3 s 3 p 3 d 3 f 2 g 2 h$ & & $4 s^{2} 4 p^{6} 4 d^{10} 5 s^{2} 5 p^{3}$ & 481 \\
\hline \hline
\end{tabular}

${ }^{\mathrm{a}}$ Standard effective core potentials and valence basis sets for $\mathrm{Sb}$ are published: the ECP46MWB aug-cc-pVQZ and ECP28MDF_aug-cc-pV5Z basis sets are from MOLPRo basis set library. The ECP46MWB_aug-cc-pVQZ basis set is the same as the SDB-aug-cc-pVQZ basis from Ref. 27. The ECP28MDF_aug-cc-pVXZ ( $X=T$ and 5) basis sets are the same as aug-cc-pVXZ-PP ( $X=\mathrm{T}$ and 5) basis sets, respectively, from the EMSL basis set library (Ref. 29). For the ECP46MWB and ECP28MDF ECPs, see Refs. 31 and 28. The corresponding aug-cc-pVXZ $\left(X=\mathrm{T}, \mathrm{Q}\right.$, and 5) basis sets were used for $\mathrm{O}$ and the $\mathrm{O} 1 s^{2}$ electrons are frozen in all the correlation calculations.

${ }^{\mathrm{b}}$ Augmented basis functions designed in this work.

${ }^{\mathrm{c}}$ Although these shells of $\mathrm{Sb}$ are accounted for by the basis sets used, they are frozen in the correlation calculations. The $\mathrm{O} 1 s^{2}$ electrons are frozen in all correlation calculations.

${ }^{\mathrm{d}}$ These $\mathrm{Sb}$ electrons are correlated. The $\mathrm{O} 2 s^{2} 2 p^{4}$ electrons are correlated in all correlation calculations.

${ }^{\mathrm{e}}$ Total numbers of contracted Gaussian functions in the basis sets for $\mathrm{SbO}_{2}$.

${ }^{\mathrm{f}}$ Only $s, p, d$, and $f$ functions (i.e., no $g$ functions).

Both are density functional studies, ${ }^{16,22}$ though one of them ${ }^{22}$ has evaluated the atomization energy of $\mathrm{SbO}_{2}$ at the HartreeFock (HF) and second-order Moller-Plesset (MP2) levels employing an optimized bent structure of $\mathrm{SbO}_{2}\left[r_{e}(\mathrm{SbO})\right.$ $=1.8849 \AA$ and $\left.\theta_{e}(\mathrm{OSbO})=120.34^{\circ}\right]$ obtained at the BP86/ $\mathrm{SV}(\mathrm{P})$ level of calculation. In the other density functional study of Ref. 16 on $\mathrm{SbO}_{2}$, the local-density approximation (LDA) functional was used and the ground electronic state was computed to have a linear structure $\left[r_{e}(\mathrm{SbO})=1.87 \AA\right.$ and $\left.\theta_{e}(\mathrm{OSbO})=180.0^{\circ}\right]$, with a bent first excited electronic state $\left[r_{e}(\mathrm{SbO})=2.01 \AA, r_{e}(\mathrm{OO})=1.51 \AA\right.$, and $\theta_{e}(\mathrm{OSbO})$ $\left.=44.1^{\circ}\right]$ calculated to be $0.48 \mathrm{eV}(11.1 \mathrm{kcal} / \mathrm{mole})$ higher in energy. In both Refs. 16 and 22, however, the symmetries of the electronic states obtained have not been reported. For Ref. 22, where only one geometrical structure was reported, it can only be assumed that the reported structure is that of the ground electronic state of $\mathrm{SbO}_{2}$. In any case, it appears that from these two DFT studies, ${ }^{16,22}$ the ground electronic state of $\mathrm{SbO}_{2}$ and its geometrical structure are not well established. In fact, these two DFT studies give contradictory results concerning the geometry of the ground electronic state of $\mathrm{SbO}_{2}$ : a bent structure from Ref. 22 and a linear structure from Ref. 16.

As the first step in a project to investigate the geometrical and electronic structures of small antimony oxides and their clusters, we have carried out DFT and high-level $a b$ initio calculations on the low-lying electronic states of $\mathrm{SbO}_{2}$ with the aim of determining unambiguously its ground electronic state and geometrical structure. In addition, reliable vertical excitation energies from the ground electronic state of $\mathrm{SbO}_{2}$ have been computed with the aim of assisting future spectral identification of $\mathrm{SbO}_{2}$ in absorption and/or laser induced fluorescence (LIF) spectra yet to be recorded on $\mathrm{SbO}_{2}$ in the gas phase.

It should be noted that the ground electronic states of the dioxides of other lighter members of the group $15 p$-block (or group VA) elements, $\mathrm{NO}_{2},{ }^{23} \mathrm{PO}_{2}$ (see, Ref. 24, and references therein), and $\mathrm{AsO}_{2}$ (see Ref. 25, and references therein), are reasonably well established. They all have $\widetilde{X}^{2} A_{1}$ states, with a bent $C_{2 v}$ structure. In this connection, the linear structure obtained for the ground electronic state of $\mathrm{SbO}_{2}$ from LDA calculations reported in Ref. 16 is clearly at odds with those of the lighter group 15 analog, and hence its reliability is doubtful. However, Ref. 16 is the only available computational study, which has reported the structures of both the ground state and an excited electronic state of $\mathrm{SbO}_{2}$. Clearly further computational investigation is required to determine the ground state of $\mathrm{SbO}_{2}$.

Lastly, to our knowledge, the only spectroscopic study published on excited electronic states of $\mathrm{SbO}_{2}$ is the chemiluminescence spectrum observed from the reaction of a molecular beam of $\mathrm{Sb}$ with the scattering gas $\mathrm{O}_{3}{ }^{26} \mathrm{~A}$ broad and structureless chemiluminescence band observed in the $650-900 \mathrm{~nm}$ (ca. $44-32 \mathrm{kcal} / \mathrm{mole}$ ) region has been assigned to emission from electronically excited $\mathrm{SbO}_{2}^{*}$, in addition to a fluorescence band observed in the $400-530 \mathrm{~nm}$ region, assigned to emission from $\mathrm{SbO}^{*}$.

\section{COMPUTATIONAL DETAILS AND THEORETICAL CONSIDERATIONS}

The basis sets employed in the present study for $\mathrm{Sb}$ are summarized in Table I. Basis sets A, B, B1, and C are published standard basis sets. ${ }^{27,28}$ Basis set $\mathrm{A}$ was obtained from the EMSL basis set library. ${ }^{29}$ Basis sets B, B1, and C are from the basis set library of the MOLPRO suite of programs. ${ }^{30}$ It should be noted that basis set B (B1) is the same as the SBD-aug-cc-pVQZ basis set of Ref. 27 (see Table I), while basis sets A and C are the same as the aug-cc-pVTZ-PP and aug-cc-pV5Z-PP basis sets, ${ }^{28}$ respectively, from the EMSL website. ${ }^{29}$ The effective core potential (ECP), ECP46MWB, of basis set B (B1) is a quasirelativistic large core ECP, which accounts for the $1 s^{2} 2 s^{2} 2 p^{6} 3 s^{2} 3 p^{6} 3 d^{10} 4 s^{2} 4 p^{6} 4 d^{10}$ core electrons of $\mathrm{Sb}$ (see Ref. 31 for the ECPs used in the present study). The ECP28MDF ECP of basis sets A, C, D, and E is a fully relativistic small core ECP, with the $4 s^{2} 4 p^{6} 4 d^{10}$ elec- 
trons of $\mathrm{Sb}$ considered together with the $5 s^{2} 5 p^{3}$ electrons as valence. However, the standard valence basis sets $\mathrm{A}$ and $\mathrm{C}$ of $\mathrm{Sb}$, which employ the ECP28MDF ECP described above, only have polarized-valence- $X$-zeta $(X=\mathrm{T}$ or 5$)$ quality functions accounting for the $5 s^{2} 5 p^{3}$ electrons of $\mathrm{Sb}$, with a single contracted function describing each of the $4 s, 4 p$, and $4 d$ outer core subshells of $\mathrm{Sb}$. In order to account for the $4 s^{2} 4 p^{6}$ and $4 d^{10}$ outer core electrons of $\mathrm{Sb}$ adequately in correlation calculations, basis sets D and E were designed in the present study based on basis set C. Tight uncontracted $3 d$ (exponents: $5.6,2.8,1.4), 2 f(2.4,1.3), 2 g(2.9,1.3)$, and $2 h(2.9$, 1.3) functions, and $3 s(8.0,4.0,2.0), 3 p(6.8,3.4,1.7), 3 d$ $(6.0,3.0,1.5), 3 f(5.6,3.0,1.4) 2 g(2.9,1.3)$, and $2 h(2.9$, 1.3) functions were augmented to basis set $\mathrm{C}$ to give basis sets $\mathrm{D}$ and $\mathrm{E}$ in order to account for the $\mathrm{Sb}$ outer core $4 d^{10}$ and $4 s^{2} 4 p^{6} 4 d^{10}$ electrons, respectively. The frozen core and correlated electrons of $\mathrm{Sb}$ associated with different basis sets used in the correlation calculations carried out in the present study are summarized in Table I, together with the total number of contracted basis functions $(\mathrm{Nb})$ for $\mathrm{SbO}_{2}$. [The corresponding aug-cc-pVXZ $(X=\mathrm{T}, \mathrm{Q}$, and 5) basis sets of $\mathrm{O}$ are used and the $\mathrm{O} 1 s^{2}$ electrons are frozen in all correlation calculations.]

First, MP2 calculations and DFT calculations employing the B3LYP, PW91PW91, and HTCH407 functionals were carried out using basis set A. Geometry optimization and harmonic vibrational frequency calculations were performed using the GAUSSIAN 03 suite of programs. ${ }^{32}$ The geometries of the linear and bent structures of $\mathrm{SbO}_{2}$ reported in Refs. 16 and 22 were used as initial guesses. These preliminary calculations are for the purpose of identifying the symmetries of the linear and bent electronic states reported previously. ${ }^{16,22}$

Based on the results obtained from the MP2 and DFT calculations, it was felt that a more thorough survey on the low-lying electronic states of $\mathrm{SbO}_{2}$ would be required. At the same time, it was decided to move on from the MP2 and DFT methods, which are based on unrestricted-spin wave functions (using GAUSSIAN 03), to methods which are based on restricted-spin wave functions (using MOLPRO), so as to avoid the problem of spin contamination associated with unrestricted-spin wave functions. Survey complete active space self-consistent field (CASSCF) geometry optimization calculations using basis set B1 were first carried out, followed by RCCSD(T) and/or CASSCF/MRCI (multireference configuration interaction) geometry optimization calculations using the larger basis sets B, C, and/or D. (A full valence active space was employed in all the CASSCF and MRCI calculations; see also footnotes c and d of Table I.) Numerical harmonic vibrational frequency calculations were also carried out on the lowest energy, bent, and linear electronic states of $\mathrm{SbO}_{2}$ at the $\mathrm{RCCSD}(\mathrm{T})$ level using basis set $\mathrm{B}$. Finally, RCCSD(T) single geometry energy calculations using basis set E were also performed at $\operatorname{RCCSD}(\mathrm{T}) / \mathrm{D}$ geometries for some low-lying states of $\mathrm{SbO}_{2}$, and the geometry of the ground state of $\mathrm{SbO}_{2}$ was optimized at the RCCSD(T)/E level of calculation. The purpose of this series of calculations is to obtain reliable adiabatic relative electronic energies of the low-lying electronic states of $\mathrm{SbO}_{2}$ and hence to determine the ground state of $\mathrm{SbO}_{2}$. In this connection, geometry optimization calculations at higher levels of theory were only carried out for a few lowest-lying electronic states.

Further calculations were carried out to obtain reliable vertical excitation energies from the ground electronic state of $\mathrm{SbO}_{2}$. The purpose of these calculations is to assist future spectral assignments of absorption and/or LIF spectra yet to be observed. In relation to assisting spectral assignments, vertical excitation energies are more relevant than adiabatic excitation energies, as the adiabatic position of a spectral band may not be identifiable because of having a small Franck-Condon factor. CASSCF, CASSCF/MRCI, and/or RCCSD $(\mathrm{T})$ calculations using basis sets B1, B; C, and/or D were carried out at appropriate optimized geometries of the ground electronic state of $\mathrm{SbO}_{2}$ in order to obtain reliable vertical excitation energies.

In the above calculations, scalar relativistic effects from $\mathrm{Sb}$ are accounted for by using the quasirelativistic (ECP46MWB) or relativistic (ECP28MDF) ECPs (see, for example, Ref. 27). The use of different valence basis sets and/or different frozen cores for $\mathrm{Sb}$ is to assess the effects of basis size extension to the complete basis set (CBS) limit and/or outer core electron correlation from $\mathrm{Sb}$, respectively, on the optimized geometrical parameters and computed relative electronic energies. The computed CASSCF and/or MRCI wave functions, and/or the computed $T_{1}$ diagnostics from the RCCSD(T) calculations would provide us information on the importance of multireference character for the low-lying electronic states of $\mathrm{SbO}_{2}$.

What remains to be considered is spin-orbit (SO) coupling among the low-lying electronic states of $\mathrm{SbO}_{2}$. Since the ground electronic state of $\mathrm{SbO}_{2}$ and the two lowest-lying excited states, which are close in energy to the ground state, as determined in the present study, have $C_{2 v}$ structures (see next section), and are therefore nondegenerate states, they do not have diagonal spin-orbit splitting. Nevertheless, offdiagonal spin-orbit interactions between states, which are close to each other in energy, may be significant for a molecule containing the heavy fourth row element Sb. Consequently, spin-orbit coupling among low-lying states of $\mathrm{SbO}_{2}$ was calculated explicitly by the state-interacting method, which employs the average-state CASSCF wave functions of the electronic states considered, as implemented in MOLPRO. Spin-orbit matrix elements and eigenstates were computed using either the Breit-Pauli (BP) operator or spin-orbit pseudopotentials (ECPs). In the former case of all-electron calculations, uncontracted $s, p$, and $d$ functions from the DZVP-DFT-orb basis sets ${ }^{33}$ (which are all-electron basis sets) were used for $\mathrm{Sb}$ and $\mathrm{O}$. In the latter case with the ECP28MWB ECP for $\mathrm{Sb}$, uncontracted $s, p$, and $d$ functions of basis set B were used. In both cases, more reliable computed electronic energies, which include dynamic electron correlation, were used for the diagonal spin-orbit matrix elements, instead of the CASSCF energies associated with the average-state CASSCF wave functions used in the spin-orbit calculations. For vertical excitation energies, the $\mathrm{CASSCF} / \mathrm{MRCI}+\mathrm{D}$ (internally contracted MRCI plus Davidson correction) energies of the states considered, obtained using basis set B, were used instead of the averagestate CASSCF energies for the diagonal spin-orbit matrix 
elements. Four states (the lowest state of each symmetry in the $C_{2 v}$ symmetry group) and eight states (the lowest two states of each symmetry) were considered separately in these calculations. In addition, the $\operatorname{RCCSD}(\mathrm{T}) / \mathrm{D}$ energies of the lowest four states were also used for the diagonal spin-orbit matrix elements in the CASSCF spin-orbit calculations using the ECP basis set. For the computed $T_{e}$ 's of the first two excited electronic states of $\mathrm{SbO}_{2}$, spin-orbit coupling was included at each RCCSD(T)/D minimum-energy geometry, using the ECP basis set mentioned above. The electronic energies of the states considered in these spin-orbit calculations were computed at the RCCSD(T)/B level at the relevant geometry and were used for the diagonal spin-orbit matrix elements. Only the lowest three states of $\mathrm{SbO}_{2}$ have been considered in these CASSCF SO calculations, as higher states are significantly higher in energy at the geometries concerned.

\section{RESULTS AND DISCUSSION}

\section{MP2 and DFT results}

First, from Table II, it can be seen that MP2 and DFT calculations give the consistent result that the lowest energy state is the ${ }^{2} A_{1}$ state with the electronic configuration of $\cdots\left(5 a_{1}\right)^{1}\left(1 b_{1}\right)^{2}\left(4 b_{2}\right)^{2}\left(1 a_{2}\right)^{2}$. However, the calculated bond angle of $137.33^{\circ}$ obtained at the MP2 level is significantly larger than those (ca. $122^{\circ}$ ) obtained from DFT calculations with the three functionals used. Second, the electronic state obtained for the linear structure is also consistent from both MP2 and DFT calculations to be a ${ }^{2} \Sigma_{g}^{+}$state. This linear state is calculated to be higher in energy than the lowest ${ }^{2} A_{1}$ state by ca. $13-21 \mathrm{kcal} /$ mole, with the computed DFT relative energies (with respect to the lowest ${ }^{2} A_{1}$ state) larger than the MP2 one. Third, with an initial geometry with a bent structure with a small bond angle of $44.1^{\circ}$ as obtained from LDA calculations of Ref. 16, MP2 geometry optimization leads to a ${ }^{2} A_{2}$ state, which has a computed PUMP2 (spin projection of unrestricted-spin MP2 energy) energy of $68.0 \mathrm{kcal} / \mathrm{mole}$ higher than the lowest ${ }^{2} A_{1}$ state. However, DFT geometry optimization calculations with this initial geometry with the three functionals used give a ${ }^{2} B_{1}$ state in all cases, and this ${ }^{2} B_{1}$ state has computed energies ranging from 24.7 to 27.9 $\mathrm{kcal} / \mathrm{mole}$ above the lowest ${ }^{2} A_{1}$ state, depending on the functional used. Comparing these results with those reported previously, it seems clear that the bent structure of Ref. 22 corresponds to the lowest energy ${ }^{2} A_{1}$ state obtained here, while the linear and bent structures of Ref. 16 correspond to the ${ }^{2} \Sigma_{g}^{+}$and ${ }^{2} B_{1}$ states obtained here, respectively. In this connection, both structures from LDA calculations of Ref. 16 are those of excited states of $\mathrm{SbO}_{2}$.

When the computed MP2 and DFT harmonic vibrational frequencies obtained in the present study are considered (see Table II), the MP2 calculations give one unrealistically large vibrational frequency for both the ${ }^{2} A_{1}$ state [the asymmetric stretching mode of $\left.2174 \mathrm{~cm}^{-1}\left(b_{2}\right)\right]$ and the ${ }^{2} \Sigma_{g}^{+}$state [the asymmetric stretching mode of $\left.2571 \mathrm{~cm}^{-1}\left(\sigma_{u}\right)\right]$, as compared with the corresponding DFT values (less than $800 \mathrm{~cm}^{-1}$; see Table II). It is noted that the computed $\left\langle S^{2}\right\rangle$ values of the unrestricted-spin Hartree-Fock (UHF) wave functions for the ${ }^{2} A_{1}$ and ${ }^{2} \Sigma_{g}^{+}$states of $\mathrm{SbO}_{2}$ obtained from the MP2 calculations are 0.853 and 0.817 , respectively, suggesting that spin contaminations in the UHF wave functions of these two doublet states from higher-spin quartet states are not negligible. In contrast, the computed $\left\langle S^{2}\right\rangle$ values from the corresponding DFT calculations are less than 0.77 in all cases. It appears that the unrealistically large computed asymmetric stretching frequencies of these two states of $\mathrm{SbO}_{2}$ obtained from the MP2 calculations are very likely due to spin contaminations in the UHF wave functions. In addition, the MP2 vibrational frequencies are all real for the ${ }^{2} \Sigma_{g}^{+}$ state, but the DFT calculations give imaginary vibrational frequencies for the doubly degenerate bending $\left(\pi_{u}\right)$ modes with all three functionals used for this state, indicating that this linear structure is a saddle point on the DFT energy surfaces. In view of the above considerations, it is concluded that the MP2 method is probably inadequate for the lowlying electronic states of $\mathrm{SbO}_{2}$. We will come back to this point, when results obtained from higher level calculations are considered.

\section{CASSCF and higher level calculations}

The results obtained from CASSCF, $\operatorname{RCCSD}(\mathrm{T})$, and CASSCF/MRCI geometry optimization calculations are summarized in Tables II and III. First, from Table II, when the results obtained from the CASSCF/B1 and $\operatorname{RCCSD}(\mathrm{T}) / \mathrm{B}$ calculations are compared, they are qualitatively, reasonably consistent with each other. This is generally the case for both the optimized geometrical parameters and computed relative electronic energies, for almost all of the electronic states of $\mathrm{SbO}_{2}$ under investigation. The only major qualitative difference is with the (2) ${ }^{2} B_{1}$ state with the $\cdots\left(4 a_{1}\right)^{2}\left(2 b_{1}\right)^{1}\left(4 b_{2}\right)^{2}\left(1 a_{2}\right)^{2}$ configuration: CASSCF/B1 geometry optimization calculations give a bond angle of $158.9^{\circ}$, but $\mathrm{RCCSD}(\mathrm{T}) / \mathrm{B}$ calculations give a linear structure, which corresponds to a ${ }^{2} \Pi_{u}$ state. Apparently, dynamic electron correlation favors a larger bond angle for this state. This seems to be also the case for the lowest energy ${ }^{2} A_{1}$ state, though to a lesser extent: CASSCF calculations give a bond angle of $117.1^{\circ}$, while $\operatorname{RCCSD}(\mathrm{T})$ calculations give a bond angle of $122.8^{\circ}$. Regarding computed relative electronic energies of the low-lying electronic states of $\mathrm{SbO}_{2}$ investigated, those obtained from CASSCF calculations, where dynamic electron correlation is lacking, have a narrower energy spread than those from $\operatorname{RCCSD}(\mathrm{T})$ calculations.. It is clear that dynamic electron correlation is important in determining the relative electronic energy separations among low-lying electronic states of $\mathrm{SbO}_{2}$.

Second, when the $\mathrm{RCCSD}(\mathrm{T}) / \mathrm{B}$ results are compared with the MP2 and DFT results, agreement for both optimized geometrical parameters and computed relative electronic energies is generally reasonably good. Nevertheless, one exception should be noted. Although the computed $\operatorname{RCCSD}(\mathrm{T}) / \mathrm{B}$ bond angle for the ${ }^{2} A_{1}$ state of $122.8^{\circ}$ is in very good agreement with DFT values of ca. $122.6^{\circ}$ obtained using the PW91PW91 and HCTH407 functionals, it is considerably smaller than the MP2 value of $137.3^{\circ}$. This supports the conclusion made above that the MP2 method is 
TABLE II. The optimized geometrical parameters $\left(r_{e}\right.$ in $\AA$ and $\theta_{e}$ in deg), computed harmonic vibrational frequencies $\left(\omega_{e}\right.$ 's in $\left.\mathrm{cm}^{-1}\right)$, and relative electronic energies ( $E_{\text {rel }}$ in $\mathrm{kcal} / \mathrm{mole}$ ) of some low-lying electronic states of $\mathrm{SbO}_{2}$ obtained at different levels of calculation. (For basis sets used, see Table I).

\begin{tabular}{|c|c|c|c|c|}
\hline & $r_{e}(\mathrm{SbO})$ & $\theta_{e}$ & $\begin{array}{l}\text { Vibrational } \\
\text { frequencies }\end{array}$ & $E_{\text {rel }}$ \\
\hline \multicolumn{5}{|c|}{ Previous studies } \\
\hline \multicolumn{5}{|l|}{$\mathrm{LDA}^{\mathrm{a}}$} \\
\hline Electronic state unknown & $2.01^{\mathrm{b}}$ & 44.1 & & $11.1^{\mathrm{b}}$ \\
\hline Electronic state unknown & 1.87 & 180.0 & & 0.0 \\
\hline \multicolumn{5}{|l|}{$\mathrm{BP} 86 / \mathrm{SV}(\mathrm{P})^{\mathrm{c}}$} \\
\hline Electronic state unknown & 1.8849 & 120.34 & & 0.0 \\
\hline \multicolumn{5}{|c|}{ Present work } \\
\hline \multicolumn{5}{|l|}{$\mathrm{MP} 2 / \mathrm{A}$} \\
\hline${ }^{2} \mathrm{~A}_{2}$ & $2.1502^{\mathrm{d}}$ & 36.78 & $457\left(a_{1}\right), 686\left(b_{2}\right), 1013\left(a_{1}\right)$ & 68.0 \\
\hline${ }^{2} \Sigma_{g}$ & 1.8243 & 180.0 & $225\left(\pi_{u}\right), 924\left(\sigma_{g}\right), 2571\left(\sigma_{u}\right)$ & 13.4 \\
\hline${ }^{2} A_{1}$ & 1.8097 & 137.33 & $225\left(a_{1}\right), 904\left(a_{1}\right), 2174\left(b_{2}\right)$ & 0.0 \\
\hline \multicolumn{5}{|c|}{ B3LYP/A } \\
\hline${ }^{2} B_{1}$ & $1.9986^{\mathrm{e}}$ & 44.41 & $513\left(a_{1}\right), 568\left(b_{2}\right), 870\left(a_{1}\right)$ & 24.7 \\
\hline${ }^{2} \Sigma_{g}$ & 1.8658 & 180.0 & $294 i\left(\pi_{u}\right), 662\left(\sigma_{u}\right), 704\left(\sigma_{g}\right)$ & 20.7 \\
\hline${ }^{2} A_{1}$ & 1.8489 & 120.98 & $206\left(a_{1}\right), 578\left(b_{2}\right), 756\left(a_{1}\right)$ & 0.0 \\
\hline \multicolumn{5}{|c|}{ PW91PW91/A } \\
\hline${ }^{2} B_{1}$ & 2.0140 & 44.41 & $496\left(a_{1}\right), 555\left(b_{2}\right), 847\left(a_{1}\right)$ & 26.4 \\
\hline${ }^{2} \Sigma_{g}$ & $1.8804^{\mathrm{f}}$ & 180.0 & $270 i\left(\pi_{u}\right), 674\left(\sigma_{g}\right), 675\left(\sigma_{u}\right)$ & 17.4 \\
\hline${ }^{2} A_{1}$ & 1.8623 & 122.54 & $199\left(a_{1}\right), 687\left(b_{2}\right), 725\left(a_{1}\right)$ & 0.0 \\
\hline \multicolumn{5}{|c|}{ НCTH407/A } \\
\hline${ }^{2} B_{1}$ & 2.0048 & 43.90 & $497\left(a_{1}\right), 562\left(b_{2}\right), 849\left(a_{1}\right)$ & 27.9 \\
\hline${ }^{2} \Sigma_{g}$ & 1.8709 & 180.0 & $279 i\left(\pi_{u}\right), 670\left(\sigma_{u}\right), 675\left(\sigma_{g}\right)$ & 18.0 \\
\hline${ }^{2} A_{1}$ & $1.8528^{\mathrm{g}}$ & 122.72 & $202\left(a_{1}\right), 680\left(b_{2}\right), 725\left(a_{1}\right)$ & 0.0 \\
\hline \multicolumn{5}{|c|}{ CASSCF/B1 } \\
\hline${ }^{2} B_{1}\left(4 a_{1}\right)^{2}\left(2 b_{1}\right)^{1}\left(4 b_{2}\right)^{2}\left(1 a_{2}\right)^{2}$ & 1.8581 & 158.91 & & 62.5 \\
\hline${ }^{2} A_{2}\left(5 a_{1}\right)^{2}\left(2 b_{1}\right)^{2}\left(3 b_{2}\right)^{2}\left(1 a_{2}\right)^{1}$ & $2.1705^{\mathrm{h}}$ & 36.06 & & 49.9 \\
\hline${ }^{2} \Sigma_{g}^{+}\left(3 \sigma_{u}\right)^{2}\left(1 \pi_{u}\right)^{4}\left(1 \pi_{g}\right)^{4}\left(4 \sigma_{g}\right)^{1}$ & 1.8802 & 180.0 & & 24.8 \\
\hline${ }^{2} B_{1}\left(5 a_{1}\right)^{2}\left(2 b_{1}\right)^{1}\left(3 b_{2}\right)^{2}\left(1 a_{2}\right)^{2}$ & $1.9860^{\mathrm{i}}$ & 46.56 & & 23.3 \\
\hline${ }^{2} A_{2}\left(5 a_{1}\right)^{2}\left(1 b_{1}\right)^{2}\left(4 b_{2}\right)^{2}\left(1 a_{2}\right)^{1}$ & 1.8965 & 100.29 & & 5.2 \\
\hline${ }^{2} B_{2}\left(5 a_{2}\right)^{1}\left(1 b_{1}\right)^{2}\left(4 b_{2}\right)^{1}\left(1 a_{2}\right)^{2}$ & 1.8778 & 89.83 & & 1.7 \\
\hline${ }^{2} A_{1}\left(5 a_{1}\right)^{1}\left(1 b_{1}\right)^{2}\left(4 b_{2}\right)^{2}\left(1 a_{2}\right)^{2}$ & 1.8516 & 117.06 & & 0.0 \\
\hline \multicolumn{5}{|c|}{$\operatorname{RCCSD}(\mathrm{T}) / \mathrm{B}$} \\
\hline${ }^{2} B_{1}\left(4 a_{1}\right)^{2}\left(2 b_{1}\right)^{1}\left(4 b_{2}\right)^{2}\left(1 a_{2}\right)^{2}$ & 1.8430 & $179.99^{\mathrm{j}}$ & & 88.0 \\
\hline${ }^{2} A_{2}\left(5 a_{1}\right)^{2}\left(2 b_{1}\right)^{2}\left(3 b_{2}\right)^{2}\left(1 a_{2}\right)^{1}$ & $2.1648^{\mathrm{k}}$ & 35.76 & & 63.4 \\
\hline${ }^{2} B_{1}\left(5 a_{1}\right)^{2}\left(2 b_{1}\right)^{1}\left(3 b_{2}\right)^{2}\left(1 a_{2}\right)^{2}$ & $1.9758^{1}$ & 45.88 & & 26.7 \\
\hline${ }^{2} \Sigma_{g}^{+}\left(3 \sigma_{u}\right)^{2}\left(1 \pi_{u}\right)^{4}\left(1 \pi_{g}\right)^{4}\left(4 \sigma_{g}\right)^{1}$ & 1.8609 & 180.0 & $233 i\left(\pi_{u}\right), 691\left(\sigma_{g}\right), 1434\left(\sigma_{u}\right)$ & 20.0 \\
\hline${ }^{2} A_{2}\left(5 a_{1}\right)^{2}\left(1 b_{1}\right)^{2}\left(4 b_{2}\right)^{2}\left(1 a_{2}\right)^{1}$ & 1.8905 & 100.96 & & 10.7 \\
\hline${ }^{2} B_{2}\left(5 a_{1}\right)^{2}\left(1 b_{1}\right)^{2}\left(4 b_{2}\right)^{1}\left(1 a_{2}\right)^{2}$ & 1.8686 & 89.13 & & 5.0 \\
\hline${ }^{2} A_{1}\left(5 a_{1}\right)^{1}\left(1 b_{1}\right)^{2}\left(4 b_{2}\right)^{2}\left(1 a_{2}\right)^{2}$ & 1.8363 & 122.81 & $205\left(a_{1}\right), 762\left(a_{1}\right), 829\left(b_{2}\right)$ & 0.0 \\
\hline
\end{tabular}

${ }^{\mathrm{a}}$ Reference 16.

${ }^{\mathrm{b}}$ The optimized $\mathrm{OO}$ bond length is $1.51 \AA$ (giving a OSbO bond angle of ca. $44.1^{\circ}$ ) and the relative energy is $0.48 \mathrm{eV}$.

${ }^{\mathrm{c}}$ Reference 22.

d The optimized $\mathrm{OO}$ bond length is $1.3570 \AA$ A.

${ }^{\mathrm{e}}$ The optimized OO bond length is $1.5106 \AA$.

${ }^{\mathrm{f}}$ The optimized OO bond length is $1.5221 \AA$.

${ }^{\mathrm{g}}$ The optimized OO bond length is $1.4990 \AA$.

${ }^{\mathrm{h}}$ The optimized OO bond length is $1.3437 \AA$.

${ }^{\mathrm{i}}$ The optimized OO bond length is $1.5698 \AA$.

${ }^{j}$ Became a linear ${ }^{2} \Pi_{u}$ state.

${ }^{\mathrm{k}}$ The optimized OO bond length is $1.3294 \AA$.

${ }^{\mathrm{l}}$ The optimized OO bond length is $1.5402 \AA$. 
TABLE III. The optimized geometrical parameters $\left(r_{e}\right.$ in $\AA$ and $\theta_{e}$ in deg) and relative electronic energies $\left(E_{\text {rel }}\right.$ in $\mathrm{kcal} / \mathrm{mole}$ ) of some low-lying electronic states of $\mathrm{SbO}_{2}$ obtained at higher levels of calculation. [See Table I for the basis set and frozen core used and the electrons included in the correlated calculations. Note that with basis set $\mathrm{B}$, the $4 s^{2} 4 p^{6} 4 d^{10}$ electrons of $\mathrm{Sb}$ are accounted for by the ECP46MWB ECP and hence are not included in the numbering of the occupied molecular orbitals. However, with the basis sets $\mathrm{C}, \mathrm{D}$, and $\mathrm{E}$, the $4 s^{2} 4 p^{6} 4 d^{10}$ electrons of $\mathrm{Sb}$ are included in the Hartree-Fock calculations and hence are included in the numbering of the occupied molecular orbitals. The major configurations, the calculated CI coefficient of the major configuration $\left(c_{0}\right)$ and the sum of the squares of the CI coefficients of the reference configurations $\left(\sum c_{\mathrm{rel}}^{2}\right)$ obtained from the MRCI calculations, and the computed $T_{1}$ diagnostics from the RCCSD(T) calculations are given in the first column for CASSCF/MRCI+D/C calculations, and RCCSD(T)/D and RCCSD(T)/E calculations, respectively.]

\begin{tabular}{|c|c|c|c|c|}
\hline $\operatorname{RCCSD}(\mathrm{T}) / \mathrm{B}$ & $r_{e}$ & $\theta_{e}$ & $E_{\text {rel }}(\mathrm{RCCSD})$ & $E_{\text {rel }}\{\operatorname{RCCSD}(\mathrm{T})\}$ \\
\hline${ }^{2} \Sigma_{g}^{+}\left(3 \sigma_{u}\right)^{2}\left(1 \pi_{u}\right)^{4}\left(1 \pi_{g}\right)^{4}\left(4 \sigma_{g}\right)^{1}$ & 1.8609 & 180.0 & 24.60 & 20.04 \\
\hline${ }^{2} A_{2}\left(5 a_{1}\right)^{2}\left(1 b_{1}\right)^{2}\left(4 b_{2}\right)^{2}\left(1 a_{2}\right)^{1}$ & 1.8905 & 100.96 & 8.67 & 10.69 \\
\hline${ }^{2} B_{2}\left(5 a_{1}\right)^{2}\left(1 b_{1}\right)^{2}\left(4 b_{2}\right)^{1}\left(1 a_{2}\right)^{2}$ & 1.8686 & 89.13 & 4.43 & 5.01 \\
\hline${ }^{2} A_{1}\left(5 a_{1}\right)^{1}\left(1 b_{1}\right)^{2}\left(4 b_{2}\right)^{2}\left(1 a_{2}\right)^{2}$ & 1.8363 & 122.81 & 0.0 & 0.0 \\
\hline $\operatorname{RCCSD}(\mathrm{T}) / \mathrm{C}$ & $r_{e}$ & $\theta_{e}$ & (RCCSD) & $\{\operatorname{RCCSD}(\mathrm{T})\}$ \\
\hline${ }^{2} \Sigma_{g}^{+}\left(1 \delta_{g}\right)^{2}\left(4 \sigma_{u}\right)^{2}\left(2 \pi_{u}\right)^{4}\left(7 \sigma_{g}\right)^{1}$ & 1.87713 & 180.0 & 23.01 & 18.62 \\
\hline${ }^{2} A_{2}^{g}\left(9 a_{1}\right)^{2}\left(3 b_{1}\right)^{2}\left(6 b_{2}\right)^{2}\left(2 a_{2}\right)^{1}$ & 1.9077 & 100.34 & 8.86 & 10.62 \\
\hline${ }^{2} B_{2}\left(9 a_{1}\right)^{2}\left(3 b_{1}\right)^{2}\left(6 b_{2}\right)^{1}\left(2 a_{2}\right)^{2}$ & 1.8843 & 88.09 & 4.16 & 4.71 \\
\hline${ }^{2} A_{1}\left(9 a_{1}\right)^{1}\left(3 b_{1}\right)^{2}\left(6 b_{2}\right)^{2}\left(2 a_{2}\right)^{2}$ & 1.8514 & 122.88 & 0.0 & 0.0 \\
\hline $\mathrm{CASSCF} / \mathrm{MRCI}+\mathrm{D} / \mathrm{C}$ & $r_{e}$ & $\theta_{e}$ & (MRCI) & $(\mathrm{MRCI}+\mathrm{D})$ \\
\hline${ }^{2} \Sigma_{g}^{+} c_{0}=0.869, \Sigma c_{\mathrm{ref}}^{2}=0.947$ & 1.8667 & 180.0 & 21.64 & 20.30 \\
\hline${ }^{2} A_{2} c_{0}=0.863, \Sigma c_{\mathrm{ref}}^{2}=0.949$ & 1.9004 & 100.47 & 8.85 & 10.02 \\
\hline${ }^{2} B_{2} c_{0}=0.869, \Sigma c_{\mathrm{ref}}^{2}=0.948$ & 1.8809 & 88.95 & 3.92 & 4.07 \\
\hline${ }^{2} A_{1} c_{0}=0.879, \Sigma c_{\mathrm{ref}}^{2}=0.948$ & 1.8379 & 120.71 & 0.0 & 0.0 \\
\hline $\operatorname{RCCSD}(\mathrm{T}) / \mathrm{D}$ & $r_{e}$ & $\theta_{e}$ & (RCCSD) & $\{\operatorname{RCCSD}(\mathrm{T})\}$ \\
\hline${ }^{2} A_{2} T_{1}=0.022$ & 1.8820 & 100.31 & 9.23 & 10.68 \\
\hline${ }^{2} B_{2} T_{1}=0.033$ & 1.8597 & 87.94 & 3.92 & 4.50 \\
\hline${ }^{2} A_{1} T_{1}=0.031$ & 1.8277 & 121.87 & 0.0 & 0.0 \\
\hline $\operatorname{RCCSD}(\mathrm{T}) / \mathrm{E} / / \mathrm{RCCSD}(\mathrm{T}) / \mathrm{D}$ & $r_{e}$ & $\theta_{e}$ & (RCCSD) & $\{\operatorname{RCCSD}(\mathrm{T})\}$ \\
\hline${ }^{2} A_{2} T_{1}=0.020$ & & & 9.20 & 10.65 \\
\hline${ }^{2} B_{2} T_{1}=0.029$ & & & 3.79 & 4.39 \\
\hline${ }^{2} A_{1} T_{1}=0.027$ & & & 0.0 & 0.0 \\
\hline $\operatorname{RCCSD}(\mathrm{T}) / \mathrm{E}$ & $r_{e}$ & $\theta_{e}$ & (RCCSD) & $\{\operatorname{RCCSD}(\mathrm{T})\}$ \\
\hline${ }^{2} A_{1} T_{1}=0.027$ & 1.8271 & 121.75 & & \\
\hline
\end{tabular}

inadequate for low-lying electronic states of $\mathrm{SbO}_{2}$. Higher order electron correlation appears to be important in determining reliably the equilibrium geometrical parameters even for the ground electronic state of $\mathrm{SbO}_{2}$. In addition, the computed CASSCF bond angle of $117.1^{\circ}$ for the lowest ${ }^{2} A_{1}$ state is much closer to the corresponding DFT and $\operatorname{RCCSD}(\mathrm{T})$ values than the MP2 value. This comparison also supports the conclusion made above that spin contamination in the UHF wave function used in the MP2 calculations has an adverse effect on the MP2 results for this state.

Third, harmonic vibrational frequencies have been computed for the lowest ${ }^{2} A_{1}$ state and also the linear ${ }^{2} \Sigma_{g}^{+}$state at the $\operatorname{RCCSD}(\mathrm{T}) / \mathrm{B}$ level of calculation. For the linear ${ }^{2} \Sigma_{g}^{+}$ state, imaginary frequencies were obtained for the doubly degenerate bending $\pi_{u}$ modes, in agreement with DFT results, but contrary to MP2 results, which have all real vibrational frequencies for this linear state as discussed above. It is concluded that the MP2 energy surface of the ${ }^{2} \Sigma_{g}^{+}$state is unreliable, similar to the conclusion made above. Although the bending region of the electronic energy surface with $C_{2 v}$ symmetry near to the linear ${ }^{2} \Sigma_{g}^{+}$state saddle point has not been investigated in detail, upon bending following the $\pi_{u}$ mode with computed RCCSD(T) and/or DFT imaginary frequencies, the ${ }^{2} \Sigma_{g}^{+}$state becomes a ${ }^{2} A_{1}$ state. Since the lowest energy ${ }^{2} A_{1}$ state is calculated to be ca. $20 \mathrm{kcal} / \mathrm{mole}$ lower in energy [at the $\operatorname{RCCSD}(\mathrm{T}) / \mathrm{B}$ level of calculation] than the ${ }^{2} \Sigma_{g}^{+}$state saddle point and there is no other ${ }^{2} A_{1}$ state nearby, it is almost certain that the ${ }^{2} \Sigma_{g}^{+}$state saddle point is the barrier of linearity of the lowest ${ }^{2} A_{1}$ state. For the lowest ${ }^{2} A_{1}$ state, the vibrational frequency of the asymmetric stretching $b_{2}$ mode computed at the RCCSD(T)/B level is $829 \mathrm{~cm}^{-1}$, which is considerably smaller than the corresponding, rather large and unrealistic, MP2 value of $2174 \mathrm{~cm}^{-1}$, and is definitely closer to the DFT values of $578-687 \mathrm{~cm}^{-1}$ (depending on the functional used) than the MP2 value. However, at the RCCSD(T)/B level, the computed symmetric stretching frequency of $762 \mathrm{~cm}^{-1}$ is smaller than the asymmetric stretching frequency of $829 \mathrm{~cm}^{-1}$, but with DFT calculations, the calculated symmetric stretching frequencies (between 725 and $756 \mathrm{~cm}^{-1}$ ) are larger than the corresponding asymmetric stretching frequencies (between 578 and $678 \mathrm{~cm}^{-1}$ ) with the three functionals used. Based on results shown in Table II, it 
is concluded that DFT results are probably more reliable than MP2 results for the low-lying electronic states of $\mathrm{SbO}_{2}$, but, from the theoretical point of view, the $\operatorname{RCCSD}(\mathrm{T})$ results should be the most reliable. In this connection, the reliability of the computed harmonic vibrational frequencies obtained from DFT calculations, particularly regarding the order/ magnitudes of the symmetric and asymmetric stretching modes, is doubtful (see also the last section).

For calculations performed at higher levels of theory, only a few low energy electronic states of $\mathrm{SbO}_{2}$ were considered and the results are summarized in Table III. It should be noted that the numbering of the molecular orbitals used in the electronic configurations given in Table III is different with different ECPs used in the calculations. Nevertheless, the electronic configurations and states obtained with the two ECPs (and all basis sets) used are consistent with each other. First, the calculated CI coefficients of the major electronic configuration of the states considered, as obtained from MRCI calculations using basis set $\mathrm{C}$, and the computed $T_{1}$ diagnostics obtained from RCCSD(T) calculations using basis sets D and E are given in Table III. Both show that multireference character is insignificant for these states at their equilibrium geometries.

Second, considering the optimized geometrical parameters obtained at different levels of calculation, those calculated using the CASSCF/MRCI+D method are generally smaller than those using the RCCSD(T) method with the same basis set C (see Table III). Since the MRCI method is not size consistent, while the RCCSD(T) method is size consistent, the RCCSD(T) results should be theoretically more reliable, particularly in the present cases, where multireference character for these states of $\mathrm{SbO}_{2}$ has been shown to be unimportant, as discussed. Therefore, we will focus on the RCCSD(T) results. Using a larger basis set and/or including the $4 d^{10}$ and/or $4 s^{2} 4 p^{6} 4 d^{10}$ electrons of $\mathrm{Sb}$ in the correlation calculation leads to a smaller bond angle for all the bent states studied. In this connection, the computed $\operatorname{RCCSD}(\mathrm{T}) / \mathrm{D}$ [or RCCSD(T)/E, for the lowest energy ${ }^{2} A_{1}$ state] bond angles are probably the most reliable and are upper limits to the true values. The maximum theoretical uncertainty associated with the best computed bond angles is probably around $1^{\circ}$ based on the results obtained in the present study. For example, it is essentially the inclusion of the $4 d^{10}$ outer core electrons of $\mathrm{Sb}$ in the correlation calculation, which reduces the equilibrium bond angle of the $\tilde{X}^{2} A_{1}$ state of $\mathrm{SbO}_{2}$ by ca. $1^{\circ}$ [the difference between the $\operatorname{RCCSD}(\mathrm{T}) / \mathrm{C}$ and $\mathrm{RCCSD}(\mathrm{T}) / \mathrm{D}$ bond angles; the inclusion of the $4 s^{2} 4 p^{6}$ electrons of $\mathrm{Sb}$ with basis set $\mathrm{E}$ has insignificantly small effects on the computed equilibrium geometrical parameters]. Regarding the optimized bond lengths, they increase from using basis set B (quadruple-zeta quality) to basis set C (quintuple-zeta quality), but decrease when the $4 d^{10}$ electrons of $\mathrm{Sb}$ are included in the correlation calculations, using basis set D. In this connection, the best calculated bond lengths are probably close to the corresponding computed RCCSD(T)/B values, with uncertainties of ca. $\pm 0.01 \AA$.

Third, considering computed relative electronic energies $\left(T_{e}\right)$ obtained at different levels of calculation, contributions from both the Davidson corrections in the MRCI+D calcu- lations and the triple excitations in the $\operatorname{RCCSD}(\mathrm{T})$ calculations to the computed relative electronic energies are generally small, but not insignificant (mostly less than $2 \mathrm{kcal} / \mathrm{mole})$. The largest contribution from triple excitations in a $\operatorname{RCCSD}(\mathrm{T})$ calculation to the computed $T_{e}$ is with the ${ }^{2} \Sigma_{g}^{+}$state [larger than $4 \mathrm{kcal} / \mathrm{mole}$ at the $\operatorname{RCCSD}(\mathrm{T})$ level with basis sets $\mathrm{B}$ and $\mathrm{C}$; see Table III]. Nevertheless, the computed $\mathrm{RCCSD}(\mathrm{T})$ and $\mathrm{MRCI}+\mathrm{D}$ relative electronic energies obtained with the same basis set $\mathrm{C}$ are almost identical, with the only exception being the ${ }^{2} \Sigma_{g}^{+}$state, where the computed $\mathrm{MRCI}+\mathrm{D} / \mathrm{C} T_{e}$ of $20.30 \mathrm{kcal} / \mathrm{mole}$ is larger than the $\operatorname{RCCSD}(\mathrm{T}) / \mathrm{C}$ value of $18.62 \mathrm{kcal} / \mathrm{mole}$ by ca. $1.7 \mathrm{kcal} / \mathrm{mole}$. If the ${ }^{2} \Sigma_{g}^{+}$state, which is a saddle point on the $\mathrm{RCCSD}(\mathrm{T}) / \mathrm{B}$ electronic energy surface, is ignored, it can be concluded that the computed $\operatorname{RCCSD}(\mathrm{T})$ and $\mathrm{CASSCF} /$ MRCI relative electronic energies obtained for the three lowest energy states of $\mathrm{SbO}_{2}\left({ }^{2} A_{2},{ }^{2} B_{2}\right.$, and $\left.{ }^{2} A_{1}\right)$ with basis sets $\mathrm{B}$ and $\mathrm{C}$ are very consistent. When outer core correlation is included in the RCCSD(T) calculations, where basis sets D and $\mathrm{E}$ were employed, it can be seen that its effects on the computed relative electronic energies of the three lowest states studied are negligibly small (less than $0.5 \mathrm{kcal} / \mathrm{mole}$ ). Based on the RCCSD(T) results obtained using basis sets B, $\mathrm{C}, \mathrm{D}$, and $\mathrm{E}$, the maximum computed uncertainties associated with the computed $T_{e}$ values of the $\widetilde{A}^{2} B_{2}$ and $\widetilde{B}^{2} A_{2}$ states of $\mathrm{SbO}_{2}$ obtained at the RCCSD(T)/E//RCCSD(T)/D level should be less than $1 \mathrm{kcal} / \mathrm{mole}$. It should be noted that calculated spin-orbit contributions to the computed electronic energies of the lowest three electronic states of $\mathrm{SbO}_{2}$ are negligibly small (a decrease of $0.6 \mathrm{~cm}^{-1}$ for the $\widetilde{X}^{2} A_{1}$ state, a decrease of $0.8 \mathrm{~cm}^{-1}$ for the $\widetilde{A}^{2} B_{2}$ state, and an increase of $0.1 \mathrm{~cm}^{-1}$ for the $\widetilde{B}^{2} A_{2}$ state).

Summarizing, the computed relative electronic energies of the three lowest energy states of $\mathrm{SbO}_{2}$ reported here are found to be very consistent at higher levels of calculation and hence are concluded to be highly reliable. In this connection, the ground electronic state of $\mathrm{SbO}_{2}$ is determined unambiguously to be the $\widetilde{X}^{2} A_{1}$ state. Nevertheless, the $T_{e}$ values of the $\widetilde{A}^{2} B_{2}$ and $\widetilde{B}^{2} A_{2}$ states of $\mathrm{SbO}_{2}$ are estimated to be 4.1 and $10.7 \mathrm{kcal} / \mathrm{mole}$ at the complete basis set (CBS) limit [taking half of the difference between the RCCSD(T)/B and $\operatorname{RCCSD}(\mathrm{T}) / \mathrm{E}$ values as the CBS correction]. The $\tilde{A}^{2} B_{2}$ state of $\mathrm{SbO}_{2}$ is thus quite close in energy to the $\widetilde{X}^{2} A_{1}$ state.

\section{Vertical excitation energies}

The computed vertical excitation energies $\left(T_{\text {vert }}\right)$ and transition dipole moments (TDMs) obtained from averagestate CASSCF calculations using basis set B1 are summarized in Table IV. The results obtained from the four-state and eight-state calculations are shown and their differences, which are small, are primarily due to their different stateaverage CASSCF wave functions. The computed TDMs suggest that electronic excitation to the (2) ${ }^{2} A_{1}$ state from the $\widetilde{X}^{2} A_{1}$ state is the strongest, followed by excitation to the (1) ${ }^{2} B_{2}$ (or $\widetilde{A}^{2} B_{2}$ ) state. These results on $\mathrm{SbO}_{2}$ are similar to those reported previously on $\mathrm{PO}_{2}{ }^{24}$ However, the (2) ${ }^{2} A_{1}$ 
TABLE IV. Computed vertical excitation energies $\left(T_{\text {vert }}\right.$ in $\left.\mathrm{kcal} / \mathrm{mole}\right)$ and transition dipole moment [TDM (the computed transition dipole moment matrix element: 〈excited state|DMX, $Y$, or $Z$ [dipole moment in the $x$, $y$, or $z$ direction (under TDM in parentheses)] $\mid$ ground state $\rangle$ ) in Debye] of some low-lying doublet states of $\mathrm{SbO}_{2}$ from the $\tilde{X}^{2} A_{1}$ state (the CASSCF averaged-state calculations of the low-lying excited states of $\mathrm{SbO}_{2}$ were carried out at the CASSCF/MRCI+D/B optimized geometry of the $\tilde{X}^{2} A_{1}$ state) by four-states [the four states are the lowest state of symmetry belonging to each irreducible representation of the $C_{2 v}$ symmetry point group (including the $\tilde{X}^{2} A_{1}$ state)] and eight-states [the eight states are the lowest two states of symmetry belonging to each irreducible representation of the $C_{2 v}$ symmetry point group (including the $\tilde{X}^{2} A_{1}$ state)] averaged-state CASSCF calculation employing basis set B1 (see Table I).

\begin{tabular}{|c|c|c|c|c|c|}
\hline \multirow{2}{*}{$\begin{array}{l}\text { CASSCF } \\
\text { States }\end{array}$} & \multirow[b]{2}{*}{ Major configuration } & \multicolumn{2}{|c|}{ Four states } & \multicolumn{2}{|c|}{ Eight states } \\
\hline & & $T_{\text {vert }}$ & TDM & $T_{\text {vert }}$ & TDM \\
\hline${ }^{2} \mathrm{~A}_{2}$ & $\left(5 a_{1}\right)^{2}\left(1 b_{1}\right)^{2}\left(4 b_{2}\right)^{2}\left(1 a_{2}\right)^{1}$ & 13.7 & 0.0 & 13.7 & 0.0 \\
\hline${ }^{2} B_{2}$ & $\left(5 a_{1}\right)^{2}\left(1 b_{1}\right)^{2}\left(4 b_{2}\right)^{1}\left(1 a_{2}\right)^{2}$ & 16.5 & $0.965(Y)$ & 16.3 & $0.829(Y)$ \\
\hline${ }^{2} B_{1}$ & $\left(5 a_{1}\right)^{2}\left(1 b_{1}\right)^{1}\left(4 b_{2}\right)^{2}\left(1 a_{2}\right)^{2}$ & 50.6 & $0.747(X)$ & 48.5 & $0.692(X)$ \\
\hline${ }^{2} A_{1}$ & $\left(4 a_{1}\right)^{1}\left(5 a_{.1}\right)^{2}\left(1 b_{1}\right)^{2}\left(4 b_{2}\right)^{2}\left(1 a_{2}\right)^{2}$ & & & 55.7 & $1.141(Z)$ \\
\hline${ }^{2} B_{2}$ & $\left(5 a_{1}\right)^{2}\left(1 b_{1}\right)^{2}\left(3 b_{2}\right)^{1}\left(4 b_{2}\right)^{2}\left(1 a_{2}\right)^{2}$ & & & 59.4 & $0.455(Y)$ \\
\hline${ }^{2} B_{1}$ & $\left(4 a_{1}\right)^{2}\left(1 b_{1}\right)^{2}\left(2 b_{1}\right)^{1}\left(4 b_{2}\right)^{2}\left(1 a_{2}\right)^{2}$ & & & 69.9 & $-0.717(X)$ \\
\hline${ }^{2} \mathrm{~A}_{2}$ & $\left(5 a_{1}\right)^{1}\left(1 b_{1}\right)^{2}\left(2 b_{1}\right)^{1}\left(4 b_{2}\right)^{1}\left(1 a_{2}\right)^{2}$ & & & 79.4 & 0.0 \\
\hline
\end{tabular}

TABLE V. Computed vertical excitation energies ( $T_{\text {vert }}$ in $\mathrm{kcal} / \mathrm{mole}$ ) of some low-lying doublet states (see Table IV for the main configurations of the electronic states) of $\mathrm{SbO}_{2}$ from the $\tilde{X}^{2} A_{1}$ state obtained at higher levels of calculation. [Calculations were carried out at the corresponding CASSCF/MRCI+D or RCCSD(T) optimized geometries of the $\widetilde{X}^{2} A_{1}$ state of $\mathrm{SbO}_{2}$. With basis B, the CASSCF/MRCI calculations have considered two roots for the state symmetries of $A_{1}, B_{1}, B_{2}$, and $A_{2}$. With basis $\mathrm{C}$, only the lowest ${ }^{2} A_{2}$ state was considered.]

\begin{tabular}{|c|c|c|c|c|c|c|c|}
\hline$T_{\text {vert }}$ & ${ }^{2} A_{2}$ & ${ }^{2} B_{2}$ & ${ }^{2} B_{1}$ & ${ }^{2} A_{1}$ & ${ }^{2} B_{2}$ & ${ }^{2} B_{1}$ & ${ }^{2} A_{2}$ \\
\hline $\mathrm{CASSCF} / \mathrm{B}$ & 17.92 & 16.98 & 53.39 & 57.32 & 62.72 & 69.17 & 78.99 \\
\hline CASSCF/MRCI/B & 19.87 & 20.14 & 51.94 & 54.31 & 60.63 & 68.32 & 81.15 \\
\hline $\mathrm{CASSCF} / \mathrm{MRCI}+\mathrm{D} / \mathrm{B}$ & 19.58 & 18.96 & 51.16 & 53.10 & 59.72 & 67.66 & 81.00 \\
\hline $\mathrm{CAS} / \mathrm{MRCI}+\mathrm{D} / \mathrm{B}+\mathrm{SO}\left(\right.$ ecp_4states $^{\mathrm{a}}{ }^{\mathrm{a}}$ & 20.16 & 18.71 & 51.16 & & & & \\
\hline $\mathrm{CAS} / \mathrm{MRCI}+\mathrm{D} / \mathrm{B}+\mathrm{SO}\left(\mathrm{ecp}_{-} 8 \text { states }\right)^{\mathrm{b}}$ & 20.15 & 18.71 & 51.16 & 53.10 & 59.72 & 67.66 & 81.01 \\
\hline $\mathrm{CAS} / \mathrm{MRCI}+\mathrm{D} / \mathrm{B}+\mathrm{SO}(\text { all_e_} 8 \text { states })^{\mathrm{c}}$ & 20.13 & 18.70 & 50.16 & 53.62 & 60.23 & 67.70 & 81.02 \\
\hline $\mathrm{CASSCF} / \mathrm{C}$ & 13.16 & 17.26 & 53.67 & 57.25 & 62.70 & 68.91 & \\
\hline $\mathrm{CASSCF} / \mathrm{MRCI} / \mathrm{C}$ & 19.17 & 18.97 & 52.36 & 54.38 & 60.72 & 68.01 & \\
\hline $\mathrm{CASSCF} / \mathrm{MRCI}+\mathrm{D} / \mathrm{C}$ & 20.79 & 19.1 & 51.66 & 53.26 & 59.89 & 67.35 & \\
\hline $\mathrm{RCCSD}^{\mathrm{d}} / \mathrm{C}$ & 19.71 & 21.10 & 55.75 & & & 66.96 & \\
\hline $\operatorname{RCCSD}(\mathrm{T}) / \mathrm{C}$ & 21.99 & 22.02 & 52.95 & & & 65.81 & \\
\hline $\mathrm{RCCSD}^{\mathrm{e}} / \mathrm{D}$ & 19.55 & 20.86 & 57.29 & & & 68.67 & \\
\hline $\operatorname{RCCSD}(T)^{\mathrm{e}} / \mathrm{D}$ & 21.48 & 21.61 & 54.36 & & & 66.69 & \\
\hline $\mathrm{RCCSD}(\mathrm{T}) / \mathrm{D}+\mathrm{SO}\left(\text { ecp_4 }_{-} \text {states }\right)^{\mathrm{f}}$ & 21.38 & 21.71 & 54.36 & & & & \\
\hline
\end{tabular}

${ }^{\mathrm{a}}$ The average-state CASSCF spin-orbit (SO) interaction calculations were carried out at the CASSCF/MRCI + D/B geometry of the $\widetilde{X}^{2} A_{1}$ state of $\mathrm{SbO}_{2}$, employing the ECP46MWB ECP and the uncontracted $s, p$, and $d$ functions of basis set $\mathrm{B}$ (the total number of basis functions used is 176; see Table $\mathrm{I}$ and text). The $\mathrm{CASSCF} / \mathrm{MRCI}+\mathrm{D} / \mathrm{B}$ energies were used for the SO diagonal matrix elements. Only the lowest doublet states of the four symmetries were considered. The $\tilde{X}^{2} A_{1}$ state of $\mathrm{SbO}_{2}$ was lowered by $0.68 \mathrm{~cm}^{-1}$ through offdiagonal SO interaction.

${ }^{b}$ As with footnote a, but with eight states, the $\widetilde{X}^{2} A_{1}$ state of $\mathrm{SbO}_{2}$ was lowered by $0.78 \mathrm{~cm}^{-1}$ through offdiagonal SO interaction.

${ }^{\mathrm{c}}$ As with footnotes a and $\mathrm{b}$, but the basis sets used are the uncontracted $s, p$, and $d$ functions of the all-electron DZVP-DFT-orb basis sets for both $\mathrm{Sb}$ and $\mathrm{O}$. The total number of basis functions used is 163 ; the $\widetilde{X}^{2} A_{1}$ state of $\mathrm{SbO}_{2}$ was lowered by $16.77 \mathrm{~cm}^{-1}$ through off-diagonal $\mathrm{SO}$ interaction.

${ }^{\mathrm{d}}$ The computed $T_{1}$ diagnostics of the four states shown are $0.0247,0.0385,0.0411$, and 0.0409 , respectively. ${ }^{\mathrm{e}}$ The computed $T_{1}$ diagnostics of the four states shown are $0.0217,0.0319,0.0343$, and 0.0338 , respectively. ${ }^{\mathrm{f}}$ As with footnote a, but the $\operatorname{RCCSD}(\mathrm{T}) / \mathrm{D}$ energies were used for the SO diagonal matrix element; the $\widetilde{X}^{2} A_{1}$ state of $\mathrm{SbO}_{2}$ was lowered by $0.6 \mathrm{~cm}^{-1}$ through off-diagonal $\mathrm{SO}$ interaction. 
$\leftarrow \tilde{X}^{2} A_{1}$ absorption or LIF band of $\mathrm{SbO}_{2}$ is expected to be in the visible region at ca. $537 \mathrm{~nm}(53 \mathrm{kcal} / \mathrm{mole}$ or $18600 \mathrm{~cm}^{-1}$; see later text and Table V), which is significantly lower in energy than the same absorption or LIF band of $\mathrm{PO}_{2}$ [with $T_{0}$ at $326 \mathrm{~nm}\left(30660 \mathrm{~cm}^{-1}\right)$; see Ref. 24, and references therein].

More reliable computed vertical excitation energies ( $T_{\text {vert }}$ 's) obtained at the MRCI+D and RCCSD(T) levels with larger basis sets are given in Table V. For an excited state of a certain spin symmetry, if its HF wave function converges to the lower state of the same spin symmetry, only CASSCF/ MRCI calculations have been carried out. In these CASSCF/ MRCI calculations, two roots were requested in order to obtain the energy of the excited state. For an excited state, which has an electronic configuration such that in the SCF procedure of the HF calculations, the configuration remains, RCCSD(T) calculations have also been carried out in addition to CASSCF/MRCI calculations [an example of this is the (2) ${ }^{2} B_{1}$ state; see Table V]. Comparing the calculated results shown in Table V, it is clear that computed CASSCF $T_{\text {vert }}$ 's, which lack dynamic electron correlation, are unreliable, as discussed when considering computed $T_{e}$ 's above. The differences in the computed CASSCF/MRCI+D $T_{\text {vert }}$ 's for the electronic states considered, between using basis sets $\mathrm{B}$ and $\mathrm{C}$, are less than $1 \mathrm{kcal} / \mathrm{mole}$. The differences in the computed $T_{\text {vert }}$ 's between using the CASSCF/MRCI+D and RCCSD(T) methods with the same basis set $\mathrm{C}$ are less than $1.5 \mathrm{kcal} / \mathrm{mole}$. The differences in the computed $T_{\text {vert }}$ 's between excluding and including $\mathrm{Sb} 4 d^{10}$ core electrons in RCCSD(T) calculations employing basis sets C and D, respectively, are less than $1.5 \mathrm{kcal} / \mathrm{mole}$ for all the electronic states considered. These comparisons suggest that the computed $T_{\text {vert }}$ values obtained at various higher levels of calculations are reasonably consistent and hence can be concluded to be reliable. The only ambiguous results are the order of the computed $T_{\text {vert }}$ 's of the (1) ${ }^{2} A_{2}$ and (1) ${ }^{2} B_{2}$ states, the two lowest excited states of $\mathrm{SbO}_{2}$. The CASSCF/MRCI+D $T_{\text {vert }}$ values with basis sets $\mathrm{B}$ and $\mathrm{C}$ place the $(1)^{2} A_{2}$ state higher in vertical excitation energy than the $(1)^{2} B_{2}$ state, but the $\operatorname{RCCSD}(\mathrm{T})$ values with basis sets $\mathrm{C}$ and $\mathrm{D}$ have a reverse order. Nevertheless, the differences in the computed $T_{\text {vert }}$ values between these two states of $\mathrm{SbO}_{2}$ are small, particularly at the RCCSD(T) level (less than $0.2 \mathrm{kcal} / \mathrm{mole}$ ). It should be noted that the computed CI coefficients (larger than 0.77 for the major configuration with basis set $\mathrm{C}$ ) and $T_{1}$ diagnostics (less than 0.035 with basis set D) obtained from the MRCI and RCCSD(T) calculations of the excited states, respectively, for vertical excitation energies from the $\tilde{X}^{2} A_{1}$ state, suggest that multireference character is not important for these excited states. In this connection, it is concluded that the computed RCCSD(T)/D $T_{\text {vert }}$ 's are the most reliable with the $T_{\text {vert }}$ to the ${ }^{2} A_{2}$ state being lower than to the ${ }^{2} B_{2}$ state by $0.13 \mathrm{kcal} / \mathrm{mole}(0.33 \mathrm{kcal} / \mathrm{mole}$ including spin-orbit coupling; see Table $\mathrm{V}$ and later text). Based on the results obtained employing basis sets $\mathrm{C}$ and $\mathrm{D}$, the uncertainties associated with the $\operatorname{RCCSD}(\mathrm{T}) T_{\text {vert }}$ 's should be less than $1.5 \mathrm{kcal} / \mathrm{mole}(0.065 \mathrm{eV})$. For excited states, where $\operatorname{RCCSD}(\mathrm{T}) T_{\text {vert }}$ 's are not available, the CASSCF/MRCI
$+\mathrm{D} / \mathrm{C} T_{\text {vert }}$ 's are the most reliable. Based on the comparison between $\mathrm{RCCSD}(\mathrm{T}) / \mathrm{D}$ and $\mathrm{CASSCF} / \mathrm{MRCI}+\mathrm{D} / \mathrm{C}$ results, the uncertainties associated with the latter values are of the order of $3.0 \mathrm{kcal} / \mathrm{mole}(0.13 \mathrm{eV})$.

The computed $T_{\text {vert }}$ values including off-diagonal spinorbit interaction are also shown in Table V. First, results obtained employing the CASSCF/MRCI+D/B energies for the diagonal matrix elements are considered. Those obtained using the BP operator with an all-electron basis set [CASSCF/MRCI+D/B+SO(all-e_8states) in Table V] and spin-orbit pseudopotentials (ECPs) with the ECP basis set ([CASSCF/MRCI+D/B+SO(ecp_4states)] and $[\mathrm{CASSCF} / \mathrm{MRCI}+\mathrm{D} / \mathrm{B}+\mathrm{SO}($ ecp_8states $)]$ ) are very similar. Also, the results obtained by considering four states (the lowest state of each symmetry) and eight states (two lowest states of each symmetry) are almost identical. Comparing the computed $T_{\text {vert }}$ 's between excluding and including spin-orbit coupling, it can be seen that off-diagonal spin-orbit effects on the computed $T_{\text {vert }}$ 's are negligibly small except for the (1) ${ }^{2} A_{2}$ and (1) ${ }^{2} B_{2}$ states, which are close in energy as mentioned above. Specifically, the computed $T_{\text {vert }}$ values of the (1) ${ }^{2} A_{2}$ state including spin-orbit coupling have increased by ca. $0.57 \mathrm{kcal} / \mathrm{mole}$, when compared with the corresponding unperturbed computed CASSCF/MRCI+D/B $T_{\text {vert }}$ values, while those of the ${ }^{2} B_{2}$ state have decreased by ca. $0.25 \mathrm{kcal} / \mathrm{mole}$. With the RCCSD(T)/D energies, which have the (1) ${ }^{2} A_{2}$ state lower in energy than the $(1)^{2} B_{2}$ state, used for the diagonal matrix elements in the average-state CASSCF spin-orbit calculations, the (1) ${ }^{2} A_{2}$ state is lowered by $0.10 \mathrm{kcal} / \mathrm{mole}$ by spin-orbit coupling, while the $(1)^{2} B_{2}$ state has increased by $0.10 \mathrm{kcal} / \mathrm{mole}$. The computed lowering in energy of the $\tilde{X}^{2} A_{1}$ state by off-diagonal spin-orbit coupling is negligibly small (less than $0.05 \mathrm{kcal} / \mathrm{mole}$; see footnotes c, d, e, and h of Table V) in all cases. Summing up, it can be concluded that effects of spin-orbit coupling on the computed $T_{\text {vert }}$ values of the low-lying electronic states of $\mathrm{SbO}_{2}$ are negligibly small.

Lastly, a few words on the only available electronic spectrum of $\mathrm{SbO}_{2}$, the chemiluminescence spectrum of Ref. 26 , seem appropriate. $\mathrm{SbO}_{2}^{*}$ may be formed in more than one excited state in the $\mathrm{Sb}+\mathrm{O}_{3}$ reaction, and it is reasonable to assume that the molecular carrier of the chemiluminescence spectrum recorded in the $650-900 \mathrm{~nm}$ region is $\mathrm{SbO}_{2}$ as suggested in Ref. 26. Moreover, it is almost certain that the excited states of $\mathrm{SbO}_{2}$ formed are vibrationally excited, and it is not unreasonable to assume that the chemiluminescence band recorded is due to emissions to the $\widetilde{X}^{2} A_{1}$ state of $\mathrm{SbO}_{2}$. In this connection, the computed vertical excitation energies from the $\tilde{X}^{2} A_{1}$ state to the excited states of $\mathrm{SbO}_{2}$ involved in the emissions may be assumed to be approximate upper limits of the high-energy onset of the chemiluminescence band at $650 \mathrm{~nm}$ (ca. $44 \mathrm{kcal} /$ mole). Based on the computed vertical excitation energies shown in Table V, the $(1)^{2} B_{1}$ and (2) ${ }^{2} A_{1}$ states with computed $T_{\text {vert }}$ values in the $52 \mathrm{kcal} / \mathrm{mole}$ (ca. $550 \mathrm{~nm}$ ) region appear to be suitable candidates for the excited states involved in the chemiluminescence spectrum reported in Ref. 26. Computed transition dipole moments between these two excited states and the $\tilde{X}^{2} A_{1}$ state also 
TABLE VI. A summary of some available geometrical parameters (in $\AA$ and deg) and vibrational frequencies (bending, symmetrical stretching, and asymmetrical stretching, in $\mathrm{cm}^{-1}$ ) of the $\widetilde{X}^{2} A_{1}$ state of the dioxides of group VA elements, and available $T_{e}$ values (in $\mathrm{kcal} / \mathrm{mole}$ ) of the $\widetilde{A}^{2} B_{2}$ state of these dioxides.

\begin{tabular}{|c|c|c|c|c|c|}
\hline$\tilde{X}^{2} A_{1}$ & Method & $r_{e}$ & $\theta_{e}$ & $\omega_{e}\left(a_{1} ; a_{1} ; b_{2}\right)$ & $T_{e}\left(\tilde{A}^{2} B_{2}\right)$ \\
\hline \multirow[t]{2}{*}{$\mathrm{NO}_{2}$} & $\mathrm{MW}^{\mathrm{a}}$ & 1.1934 & 134.1 & & \\
\hline & $\mathrm{LIF} / \mathrm{DF}^{\mathrm{b}}$ & & & $\begin{array}{l}758.636 ; 1347.53 \\
1669.02\end{array}$ & 27.9 \\
\hline \multirow[t]{5}{*}{$\mathrm{PO}_{2}$} & $\begin{array}{l}\text { LMR/MW; }{ }^{\mathrm{c}} r_{0} \text {, average } \omega \\
\text { IR laser }^{\mathrm{d}} \\
\text { LLF/DF }^{\mathrm{e}}\end{array}$ & 1.4665 & 135.3 & $\begin{array}{l}377 ; 1090 ; 1278 \\
\ldots \ldots \cdot 1327.53452(69) \\
397.3(43) ; 1075.4(50) \cdot \ldots\end{array}$ & \\
\hline & $\mathrm{MRDCI} / \mathrm{TZ}+2 d+\mathrm{R}^{\mathrm{f}}$ & 1.464 & 135.14 & $389 ; 1052 ; 1338$ & 14.45 \\
\hline & $\mathrm{MP} 2 / 6-311 \mathrm{G}(2 d)^{\mathrm{g}}$ & 1.478 & 136.6 & $403 ; 1085 ; 1486$ & 32.0 \\
\hline & $\begin{array}{l}\text { RCCSD(T)/aug-cc- } \\
\text { pVQZ }^{\text {g }}\end{array}$ & 1.474 & 134.8 & $384 ; 1069 ; \cdots$ & \\
\hline & $\begin{array}{l}\text { RCCSD(T)/aug-cc- } \\
\text { pVQZ }^{\text {h }}\end{array}$ & 1.474 & 134.8 & $390 ; 1073 ; 1349$ & \\
\hline $\mathrm{AsO}_{2}$ & $\mathrm{MP} 2 /[6 s 4 p 2 d],[4 s 2 p 1 d]^{\mathrm{i}}$ & 1.630 & 135 & & \\
\hline \multirow[t]{3}{*}{$\mathrm{SbO}_{2}$} & $\operatorname{RCCSD}(\mathrm{T}) / \mathrm{B}$ & 1.8363 & 122.81 & $205 ; 762 ; 829$ & 5.0 \\
\hline & $\operatorname{RCCSD}(\mathrm{T}) / \mathrm{E}$ & 1.8271 & 121.75 & & 4.38 \\
\hline & CBS & & & & 4.1 \\
\hline
\end{tabular}

${ }^{\mathrm{a}}$ References 23 and 46 .

${ }^{\mathrm{b}}$ References 37 and 38 .

${ }^{\mathrm{c}}$ Reference 47.

${ }^{\mathrm{d}}$ Reference 48.

${ }^{\mathrm{e}}$ Reference 45 .

${ }^{\mathrm{f}}$ Reference 41 .

${ }^{g}$ Reference 24

${ }^{\mathrm{h}}$ Reference 49 .

${ }^{\mathrm{i}}$ Reference 34. suggest that emissions from the former states to the $\tilde{X}^{2} A_{1}$ state should have significant intensities (see Table IV). In conclusion, it is not inconsistent, based on ab initio results obtained in the present study, that the chemiluminescence spectrum recorded in the $650-900 \mathrm{~nm}$ region is due to $\mathrm{SbO}_{2}^{*}$. However, further spectroscopic and computational studies will be required to firmly identify the molecular carrier of, and the electronic and vibrational states involved in, the chemiluminescence spectrum recorded in the $650-900 \mathrm{~nm}$ region resulting from the reaction between $\mathrm{Sb}$ and $\mathrm{O}_{3}$ in the gas phase. ${ }^{26}$

\section{CONCLUDING REMARKS}

DFT and high-level $a b$ initio calculations have been carried out on some low-lying electronic states of $\mathrm{SbO}_{2}$. These calculations have clarified the uncertainty associated with the geometrical structure of the ground electronic state of $\mathrm{SbO}_{2}$ reported previously. ${ }^{16,22}$ The ground state of $\mathrm{SbO}_{2}$ has been unambiguously established to be the $\tilde{X}^{2} A_{1}$ state with a $C_{2 v}$ structure, similar to the dioxides of other lighter group 15 elements. In addition, a number of low-lying electronic states of $\mathrm{SbO}_{2}$ have been characterized. Among them, it should be noted that the $\tilde{A}^{2} B_{2}$ state of $\mathrm{SbO}_{2}$ has a best computed $T_{e}$ of only $4.1 \mathrm{kcal} / \mathrm{mole}\left(1434 \mathrm{~cm}^{-1}\right.$, at the CBS limit). The closeness in energy of the $\widetilde{A}^{2} B_{2}$ state to the $\widetilde{X}^{2} A_{1}$ state will contribute to the thermodynamic properties of $\mathrm{SbO}_{2}$.

Reliable vertical excitation energies from the $\widetilde{X}^{2} A_{1}$ state to the low-lying electronic states of $\mathrm{SbO}_{2}$ have been computed. In addition, transition dipole moments (TDMs) between the $\tilde{X}^{2} A_{1}$ state and low-lying excited states of $\mathrm{SbO}_{2}$ have been calculated. These computed TDMs suggest that the strongest electronic transition between the $\tilde{X}^{2} A_{1}$ state of $\mathrm{SbO}_{2}$ and low-lying excited states is the (2) ${ }^{2} A_{1} \leftarrow \tilde{X}^{2} A_{1}$ transition, similar to a conclusion made in our previous combined $a b$ initio/Franck-Condon study on the LIF and single vibronic level emission spectra of $\mathrm{PO}_{2} \cdot{ }^{24}$ However, the computed vertical excitation energy of this transition of $\mathrm{SbO}_{2}$ obtained in the present study suggests that the absorption/ LIF band of this transition should be in the visible region, significantly lower in energy than the same transition of $\mathrm{PO}_{2}$, which is in the ultraviolet region. The calculated results reported here will be useful to assist interpretation of the absorption and/or LIF spectra of $\mathrm{SbO}_{2}$ which are/is yet to be recorded.

A summary of some available geometrical parameters and vibrational frequencies of the $\widetilde{X}^{2} A_{1}$ state of the dioxides of the group 15 (or VA) elements and available $T_{e}$ values of the $\widetilde{A}^{2} B_{2}$ state of these dioxides are given in Table VI. To our knowledge, there are no such data, theoretical or experimental, available for $\mathrm{BiO}_{2}$, the heaviest dioxide of the series. For $\mathrm{AsO}_{2}$, the optimized geometrical parameters of the $\tilde{X}^{2} A_{1}$ state have been reported at the MP2 level using the $[6 s 4 p 2 d]$ and $[4 s 2 p 1 d]$ basis sets for As and $\mathrm{O}$, respectively. ${ }^{34} \mathrm{In}$ addition, a recent computational study on cyclic $\mathrm{AsO}_{2}$ and $\mathrm{AsS}_{2}$ systems has reported optimized geometrical parameters and harmonic vibrational frequencies of the $\tilde{A}^{2} B_{2}$ state, and also other cyclic excited states of $\mathrm{AsO}_{2}$ obtained employing the MP2 and DFT (with different functionals) methods. ${ }^{25}$ However, although the relative energies of these cyclic states were calculated at higher levels of theory than the MP2 level, and a lower ${ }^{2} A_{1}$ state, which corresponds to the $\widetilde{X}^{2} A_{1}$ state of $\mathrm{AsO}_{2}$ reported in Ref. 34, was also mentioned in Ref. 25, the 
relative energy of the $\tilde{A}^{2} B_{2}$ state with respect to the $\tilde{X}^{2} A_{1}$ state of $\mathrm{AsO}_{2}$ has not been reported in Ref. 25. Consequently, the $T_{e}$ value of the $\widetilde{A}^{2} B_{2}$ states of $\mathrm{AsO}_{2}$ is unavailable. The authors of Ref. 25 have considered the $\widetilde{A}^{2} B_{2}$ state of $\mathrm{AsO}_{2}$ as the ground state of a cyclic $\mathrm{AsO}_{2}$ rather than as an excited state of $\mathrm{AsO}_{2}$. For $\mathrm{NO}_{2}$ and $\mathrm{PO}_{2}$, both theoretical and experimental geometrical parameters and vibrational frequencies are available (see Table VI). For the $T_{e}$ of the $\widetilde{A}^{2} B_{2}$ state of $\mathrm{NO}_{2}$, although there is significant vibronic interaction between the $\tilde{X}^{2} A_{1}$ and $\widetilde{A}^{2} B_{2}$ states near the conical intersection of the two electronic surfaces resulting in chaotic behavior being observed in the $10000 \mathrm{~cm}^{-1}$ region of the absorption and LIF spectra of $\mathrm{NO}_{2}$ (see Refs. 35 and 36, and references therein), the $T_{e}$ value of the $\widetilde{A}^{2} B_{2}$ state has been established to be $9760 \pm 100 \mathrm{~cm}^{-1},{ }^{37}\left[27.9 \mathrm{kcal} / \mathrm{mole} ; T_{0}\right.$ $=9734 \mathrm{~cm}^{-1}$ (Ref. 38)]. For the $T_{e}$ of the $\widetilde{A}^{2} B_{2}$ state of $\mathrm{PO}_{2}$, however, only theoretical values are available (see Table VI).

Comparing the available equilibrium geometrical parameters of the $\widetilde{X}^{2} A_{1}$ state of the dioxides of the group 15 elements, it is noted that the bond angles for $\mathrm{NO}_{2}, \mathrm{PO}_{2}$, and $\mathrm{AsO}_{2}$ have very similar values, ranging between $134^{\circ}$ and $135^{\circ}$, but $\mathrm{SbO}_{2}$ has a significantly smaller bond angle of $121.8^{\circ}$ (see Table VI). This may be compared with some group 16 (VIA) analog, $\mathrm{SO}_{2}, \mathrm{SeO}_{2}$, and $\mathrm{TeO}_{2}$, which have bond angles of $119.5^{\circ},{ }^{23} 113.83^{\circ},{ }^{39}$ and $111.15^{\circ}[\operatorname{RCCSD}(\mathrm{T}) /$ ECP46MWB-aug-cc-pV5Z, aug-cc-pV5Z value from Ref. 40], respectively. In the case of the dioxides of the group 16 elements, the significant decrease of the bond angle occurs between $\mathrm{SO}_{2}$ and $\mathrm{SeO}_{2}$. The smaller magnitude of the decrease in the bond angle between $\mathrm{SO}_{2}$ and $\mathrm{SeO}_{2}$ (ca. $6^{\circ}$ ) than that between $\mathrm{AsO}_{2}$ and $\mathrm{SbO}_{2}$ (ca. $13^{\circ}$ ) is almost certainly due to the extra valence electron in the group 16 elements and the already smaller angle in the lighter members of the group 16 dioxides than those of group 15 dioxides. In this connection, it will be interesting to see how far the bond angle would decrease with $\mathrm{BiO}_{2}$.

Comparing available vibrational frequencies of the dioxides of the group 15 elements, as shown in Table VI, the symmetric stretching frequency is smaller than the asymmetric stretching frequency for the $\tilde{X}^{2} A_{1}$ state of both $\mathrm{NO}_{2}$ and $\mathrm{PO}_{2}$. The computed RCCSD(T)/B vibrational frequencies of $\mathrm{SbO}_{2}$ reported here (see Table II) behave in the same way as the lighter members of the series with the symmetric stretching frequency being smaller than the asymmetric stretching frequency. This comparison suggests that the pattern is the same over the whole series. In this connection, the computed DFT vibrational frequencies of $\mathrm{SbO}_{2}$, which give larger values for the symmetric stretching mode than the asymmetric stretching mode (see Table II), are almost certainly unreliable, as concluded above.

Another point of interest from the comparison of the data shown in Table VI is the trend in the separations between the $\widetilde{A}^{2} B_{2}$ and $\widetilde{X}^{2} A_{1}$ states, i.e., $T_{e}$ of the $\widetilde{A}^{2} B_{2}$ state of the dioxides of the group 15 elements. Although the $T_{e}$ value of the $\widetilde{A}^{2} B_{2}$ state for $\mathrm{AsO}_{2}$ is not available, it can be seen that the separations between the $\widetilde{A}^{2} B_{2}$ and $\widetilde{X}^{2} A_{1}$ states decrease from $\mathrm{NO}_{2}$ to $\mathrm{SbO}_{2}$. In this connection, it seems clear that the MP2/6-311+G(2d) $T_{e}\left(\tilde{A}^{2} B_{2}\right)$ value of $32.0 \mathrm{kcal} / \mathrm{mole}$ from Ref. 24 for $\mathrm{PO}_{2}$ is almost certainly not as reliable as the MRDCI/TZ $+2 d+\mathrm{R}$ value of $14.45 \mathrm{kcal} / \mathrm{mole}^{41}$ (It is noted that the UHF wave function of the $\widetilde{A}^{2} B_{2}$ state of $\mathrm{PO}_{2}$ used in the MP2 calculation of Ref. 24 has a computed $\left\langle S^{2}\right\rangle$ value of 0.809 , suggesting that spin contamination from higher-spin quartet states may have affected the MP2 results.) Following the trend, as shown in Table VI, it is expected that the $T_{e}\left(\widetilde{A}^{2} B_{2}\right)$ value of $\mathrm{AsO}_{2}$ should lie between 14.45 and $4.1 \mathrm{kcal} / \mathrm{mole}$. In addition, the $T_{e}\left(\widetilde{A}^{2} B_{2}\right)$ value of $\mathrm{BiO}_{2}$ should be smaller than $4 \mathrm{kcal} / \mathrm{mole}$, and there is a possibility that for $\mathrm{BiO}_{2}$ the ground state may be a ${ }^{2} B_{2}$ state rather than a ${ }^{2} A_{1}$ state.

Regarding the appropriate computational methods to be used in studying the low-lying electronic states of $\mathrm{SbO}_{2}$ and related systems, such as $\mathrm{AsO}_{2}$ and $\mathrm{BiO}_{2}$, the results obtained from the present study shows that both the DFT (with some most commonly used functionals) and MP2 methods are inadequate at least in some aspects. Higher level methods, such as the RCCSD(T) and/or CASSCF/MRCI methods, should be employed in order to obtain reliable results. Regarding the requirements on the basis set to be used, a large core ECP, such as the ECP48MWB, with a quadruple-zeta quality valence basis set for $\mathrm{Sb}$ has been shown to be reasonably adequate. In addition, contributions from both outer core correlation and spin-orbit coupling have been found to be small.

Lastly, preliminary calculations on low-lying quartet states of $\mathrm{SbO}_{2}$ have been carried out and they suggest that the lowest quartet state is a ${ }^{4} A_{1}$ state, which is computed to be ca. $54 \mathrm{kcal} / \mathrm{mole}$ above the $\tilde{X}^{2} A_{1}$ state at the $\mathrm{CASSCF} / \mathrm{MRCI}+\mathrm{D} / \mathrm{B}$ level of calculation. It should be noted that spin-orbit interaction between doublet and quartet states can be significant, if the states involved are close in energy. However, in relation to the few lowest-lying doublet states considered in the present study, low-lying quartet states of $\mathrm{SbO}_{2}$, which are considerably higher in energy, should not affect the energy positions of these doublet states significantly via spin-orbit interaction. A full report of the investigation on low-lying quartet states of $\mathrm{SbO}_{2}$ and also low-lying singlet and triplet states of $\mathrm{SbO}_{2}^{+}$is forthcoming. On the experimental front, we call for spectroscopic studies on $\mathrm{SbO}_{2}$ (and/or similar systems, such as $\mathrm{AsO}_{2}$ and $\mathrm{BiO}_{2}$ ) to be carried out, for example, by gas phase supersonic jetcooled LIF spectroscopy. $\mathrm{SbO}_{2}$ may be prepared in the gas phase for spectroscopic study by pyrolysis (as used in the LIF studies of $\mathrm{TeO}_{2}$ (Ref. 42) and $\mathrm{SeO}_{2},{ }^{43}$ though a multistage oven would probably be required) or laser ablation (see, for example, Ref. 44) of solid antimony oxide, photolysis or electric discharge of some suitable precursors [e.g., $\mathrm{SbCl}_{3}$ or $\mathrm{SbCl}_{5}+\mathrm{O}_{2}$; the photolysis of a mixture of $\mathrm{PCl}_{3}$ and $\mathrm{O}_{2}$ molecules was employed in the LIF study of $\mathrm{PO}_{2}$ (Ref. 45)], or some suitable reactions between $\mathrm{Sb}$ atoms or small clusters (produced by laser ablation or pyrolysis) and an oxidizing agent (e.g., $\mathrm{O}$ atom produced in a microwave discharge or $\mathrm{O}_{3}$ in the throat of a supersonic nozzle). 


\section{ACKNOWLEDGMENTS}

The authors are grateful to the Research Committee of the Hong Kong Polytechnic University of HKSAR (Grant No. G-YD82 and No. G-YE06) and the Research Grant Council (RGC) of the Hong Kong Special Administrative Region (HKSAR, Grant No. AoE/B-10/1 and No. PolyU 5003/04P). Support from the EPSRC (UK) National Service for Computational Chemistry Software is also acknowledged.

${ }^{1}$ M. Kinne, T. M. Bernhardt, B. Kaiser, and K. Rademann, Int. J. Mass Spectrom. Ion Process. 167/168, 161 (1997).

${ }^{2}$ B. Kaiser, T. M. Bernhardt, M. Kinne, and K. Rademann, Int. J. Mass. Spectrom. 177, L5 (1998).

${ }^{3}$ B. Kaiser, T. M. Bernhardt, M. Kinne, K. Rademann, and A. Heidenreich, J. Chem. Phys. 110, 1437 (1999).

${ }^{4}$ J. Optiz-Coutureau, A. Fielicke, B. Kaiser, and K. Rademann, Phys. Chem. Chem. Phys. 3, 3034 (2001).

${ }^{5}$ T. Fujiwara, A. Iizuka, K. Sato, and Y. Yamada, Int. J. Mass. Spectrom. 242, 57 (2005).

${ }^{6}$ G. Centi and S. Perathoner, Appl. Catal., A 124, 317 (1995).

${ }^{7}$ S. H. Taylor, J. S. J. Hargreaves, G. J. Hutchings, and R. W. Joyner, Appl. Catal., A 126, 287 (1995).

${ }^{8}$ G. Mestl, P. B. Ruiz, B. Delmon, and H. Knozinger, J. Phys. Chem. 98, 11283 (1994).

${ }^{9}$ S. J. Gilliam, J. O. Jensen, A. Banerjee, D. Zeroka, S. J. Kirkby, and C. N. Merrow, Spectrochim. Acta, Part A 60, 423 (2004).

${ }^{10}$ C. Tian, H. Wang, X. Liu, Z. Ma, H. Guo, and J. Xu, J. Appl. Polym. Sci. 89, 3137 (2003).

${ }^{11}$ E. Jakab, Md.A. Uddin, T. Bhaskar, and Y. Sakata, J. Anal. Appl. Pyrolysis 68-69, 83 (2003).

${ }^{12}$ J.-P. Gibert, J.-M. Lopez Cuesta, A. Bergeret, and A. Crespy, Polym. Degrad. Stab. 67, 437 (2000).

${ }^{13}$ M. Zanetti, G. Camino, G. Canavese, A. B. Morgan, F. J. Lamelas, and C. A. Wilkie, Chem. Mater. 14, 189 (2002).

${ }^{14}$ S. Wang, Y. Hu, R. Zong, Y. Tang, Z. Chen, and W. Fan, Appl. Clay Sci. 25, 49 (2004).

${ }^{15}$ R. G. Egdell, M. H. Palmer, and R. H. Findlay, Inorg. Chem. 19, 1314 (1980).

${ }^{16}$ B. V. Reddy and P. Jena, Chem. Phys. Lett. 288, 253 (1998).

${ }^{17}$ A. B. Alekseyev, H.-P. Liebermaann, R. J. Buenker, and G. Hirsch, J. Chem. Phys. 102, 2539 (1995).

${ }^{18}$ S. Koseki, M. S. Gordon, M. W. Schmidt, and N. Matsunaga, J. Phys. Chem. 99, 12764 (1995)

${ }^{19}$ T. R. Cundari and E. W. Moody, J. Mol. Struct.: THEOCHEM 425, 43 (1998).

${ }^{20}$ G. Igel-mann, C. Feller, H.-J. Flad, A. Savin, H. Stoll, and H. Preuss, Mol. Phys. 68, 209 (1989).

${ }^{21}$ E. van Lenthe and E. J. Baerends, J. Comput. Chem. 24, 1142 (2003).
${ }^{22}$ F. Weigend and R. Ahlrichs, Phys. Chem. Chem. Phys. 7, 3297 (2005).

${ }^{23}$ G. Herzberg, Electronic Spectra and Electronic Structure of Polyatomic Molecules, Molecular Spectra and Molecular Structure Vol. III (Van Nostrand, Toronto, 1967).

${ }^{24}$ E. P. F. Lee, D. K. W. Mok, J. M. Dyke, and F.-T. Chau, J. Phys. Chem. A 106, 10130 (2002).

${ }^{25}$ L. Sun, Y. Bu, and S. Yan, Chem. Phys. Lett. 387, 160 (2004).

${ }^{26}$ R. P. Kempf and J. M. Parson, J. Chem. Phys. 108, 7595 (1998).

${ }^{27}$ J. M. L. Martin and J. Sundermann, J. Chem. Phys. 114, 3408 (2001).

${ }^{28}$ K. A. Peterson, J. Chem. Phys. 119, 11099 (2003).

${ }^{29}$ http://www.emsl.pnl.gov/forms/basisform.html (The Extensible Computational Chemistry Environment Basis Set Database, Version 02/25/04, as developed and distributed by the Molecular Science Computing Facility, Environmental and Molecular Sciences Laboratory which is part of the Pacific Northwest Laboratory, P.O. Box 999, Richland, Washington 99352, USA, and funded by the U.S. Department of Energy. The pacific Northwest Laboratory is a multiprogram laboratory operated by Battelle Memorial Institute for the U.S. Department of Energy under Contract No. DE-AC06-76RLO 1830. Contact Karen Schuchardt for further information.)

${ }^{30}$ H.-J. Wernen, P. J. Knowles, M. Schütz et al., MOLPRo, a package of $a b$ initio programs.

${ }^{31} \mathrm{http} / / / \mathrm{www}$. theochem.uni-stuttgart.de/cgi-bin/pe.sh?References

${ }^{32}$ M. J. Frisch, G. W. Trucks, H. B. Schlegel et al., GAussian 03, Revision B.01, Gaussian, Inc., Pittsburgh, PA, 2003.

${ }^{33}$ N. Godbout, D. R. Salahub, J. Andzelm, and E. Wimmer, Can. J. Chem. 70, 560 (1992).

${ }^{34}$ L. B. Knight, Jr., G. C. Jones, G. M. King, and R. M. Babb, J. Chem. Phys. 103, 497 (1995).

${ }^{35}$ R. Georges, A. Delon, and R. Jost, J. Chem. Phys. 103, 1732 (1995).

${ }^{36}$ E. Leonardi and C. Petrongolo, J. Chem. Phys. 106, 10066 (1997).

${ }^{37}$ A. Delon and R. Jost, J. Chem. Phys. 95, 5686 (1991).

${ }^{38}$ C. A. Biesheuvel, J. Bulthuis, M. H. M. Janssen, S. Stolte, and J. G. Snijders, J. Chem. Phys. 109, 9701 (1998).

${ }^{39}$ H. Takeo, E. Hirota, and Y. Morino, J. Mol. Spectrosc. 34, 370 (1970).

${ }^{40}$ E. P. F. Lee, D. K. W. Mok, F.-T. Chau, and J. M. Dyke, J. Chem. Phys. 121, 2962 (2004)

${ }^{41}$ Z.-L. Cai, G. Hirsch, and R. J. Buenker, Chem. Phys. Lett. 255, 350 (1996).

${ }^{42}$ D. F. Hullah and J. M. Brown, J. Mol. Spectrosc. 200, 261 (2000).

${ }^{43}$ S. A. Crowther and J. M. Brown, J. Mol. Spectrosc. 225, 196 (2004).

${ }^{44}$ K. Schofield, J. Phys. Chem. A 110, 6938 (2006).

${ }^{45}$ J. Lei, A. Teslja, B. Nizamov, and P. J. Dagdigian, J. Phys. Chem. A 105, 7828 (2001).

${ }^{46}$ G. R. Bird, J. Baird, A. W. Jache, J. A. Hodheson, R. F. Curl, Jr., A. C. Knukle, J. W. Bransford, J. Rastrup-Andrrsen, and J. Rosenthal, J. Chem. Phys. 40, 3378 (1964)

${ }^{47}$ K. Kawaguchi, S. Saiti, E. Hirota, and N. Ohashi, J. Chem. Phys. 82, 4893 (1985)

${ }^{48}$ H. B. Qian, P. B. Davie, I. K. Ahmad, and P. A. Hamilton, Chem. Phys. Lett. 235, 255 (1995).

${ }^{49}$ J. S. Francisco, J. Chem. Phys. 117, 3190 (2002). 